\title{
The inefficiency of Bitcoin revisited: A high-frequency analysis with alternative currencies
}

\author{
Ahmet Sensoy \\ Faculty of Business Administration, Bilkent University, Ankara 06800, Turkey
}

\section{A R T I C L E I N F O}

\section{Keywords:}

Efficient market hypothesis (EMH)

Bitcoin

Permutation entropy

JEL classification:

C53

G14

G15

\begin{abstract}
A B S T R A C T
We compare the time-varying weak-form efficiency of Bitcoin prices in terms of US dollars (BTCUSD) and euro (BTCEUR) at a high-frequency level by using permutation entropy. We find that BTCUSD and BTCEUR markets have become more informationally efficient at the intraday level since the beginning of 2016, and BTCUSD market is slightly more efficient than BTCEUR market in the sample period. We also find that higher the frequency, lower the pricing efficiency is. Finally, liquidity (volatility) has a significant positive (negative) effect on the informational efficiency of Bitcoin prices.
\end{abstract}

\section{Introduction}

Since the first time it was introduced by Nakamoto (2008), Bitcoin has received considerable attention among policymakers, investors and regulators. Due to its unique nature, Bitcoin can be traded any time online and exchanged into major currencies at a low cost (Fry and Cheah, 2015). The market for Bitcoin, which was created in 2009 but active trading started only in 2013, is rather young. However, its innovative features, simplicity, transparency; and the late exponential growth in its value made Bitcoin more popular than ever.

One of the key issues that is of interest to market participants is whether the pricing behaviour of Bitcoin is predictable, which would be inconsistent with the Efficient Market Hypotheses (Fama, 1970). In the past couple of years, a few academics have produced significant results on this subject via tests on the weak and semi-strong form of EMH. For instance, Urquhart (2016) uses many tests to analyse the efficiency of Bitcoin market and concludes that it becomes more efficient in the latter time sample. Nadaraj and Chu (2017) utilize eight different tests on an odd integer power transformation of Bitcoin returns and show that returns are weakly efficient. Bariviera (2017) studies the dynamics of long-range dependence properties of the Bitcoin price and finds a trend towards efficiency via Hurst exponent analysis. Similar results are found by Tiwari et al. (2018). On the other hand, using daily data with a Hurst exponent analysis, Yonghong et al. (2018) show that the Bitcoin market does not become more efficient over time. From a different perspective, Al-Yahyaee et al. (2018) compare the efficiency of Bitcoin with other assets such as gold, stock and currency. They show that Bitcoin market is the least efficient. ${ }^{1}$

Whether it is a static or a dynamic analysis, the above-mentioned studies use daily data to perform their main empirical tests. However, in today's modern financial markets, computers took over the trading scene through algorithmic (especially high-frequency) trading activities. These automated computers are programmed to take certain actions in response to varying market data in real time. The estimated percentage of the algorithmic trading volume in the total volume in US equities was $85 \%$ in 2012 , where the

\footnotetext{
E-mail addresses: ahmet.sensoy@bilkent.edu.tr, ahmets@fen.bilkent.edu.tr.

${ }^{1}$ While these studies tested the weak-form of EMH, some others tested the semi-strong form of EMH. For example, see Demir et al. (2018), Vidal-Tomas and Ibanez (2018), Panagiotidis et al. (2018) and Corbet et al. (2018).
} 
big part of this value was attributed to high frequency trading strategies (Glantz and Kissell, 2013). Today, several Bitcoin exchanges provide algorithmic trading platforms to their customers which makes it essential to analyse the Bitcoin market efficiency at the intraday level. In this study, we aim to fill this gap by testing the weak-form efficiency of Bitcoin markets through high frequency returns, which has not been covered by previous studies as far as we know.

In addition to the data frequency, we also test Bitcoin's pricing efficiency in terms of not only US dollar (BTCUSD) but also euro (BTCEUR), which is the second most traded currency in the world after US dollar. In this way, we will be able to see if it is possible to create more profitable strategies by using an alternative currency.

Previous studies use Bitcoin price data from a single exchange or a weighted price index from several exchanges. In this study, we use a tick-by-tick dataset that comes from all exchanges where USD and/or EUR can be directly used in Bitcoin trading, which is a massive extension of the previous datasets.

Our methodological framework also differs from prior studies on the weak-form efficiency of the Bitcoin markets in two ways. First, prior studies generally estimate a fixed level of market efficiency for the entire sample period. In contrast, we employ a timevarying approach by using rolling samples, giving us the flexibility of not being forced to impose cut-off dates which are usually subject to criticism in empirical studies. Second, we employ a relatively new methodology, permutation entropy, introduced by Bandt and Pompe (2002). This methodology has not been used in this context before and has several advantages over the common methodologies as explained in Section 2.

Our findings can be summarized as follows: (i) BTCUSD and BTCEUR markets have become more informationally efficient at the intraday level since the beginning of 2016, however this improvement has a cyclical pattern for BTCUSD, whereas it is a gradual increase for BTCEUR; (ii) BTCUSD market is slightly more efficient than BTCEUR market at the intraday level in the sample period; (iii) higher the frequency, lower the pricing efficiency is; and (iv) liquidity (volatility) has a significant positive (negative) effect on the informational efficiency of Bitcoin prices.

\section{Methodology}

Permutation entropy considers market efficiency as a dependency concept and translates the problem of dependency into a symbolic dynamic. A special entropy measure is associated with these symbols to test the dependency in the time series. This approach has four advantages as explained by Sensoy et al. (2015). First, the measure depends only on ordinal patterns of time series, therefore it is unaffected by the data's volatility and can detect non-linear temporal dependencies in contrast to autocorrelation. Second, the approach is model free (no assumption on distribution) hence it has a general applicability. Third, no moment is required to apply the methodology to time series. Importance of this comes from the stylized fact that asset returns are non-normally distributed and, for some distributions such as the Pareto distribution, the variance is infinite (Rachev et al., 2005). Finally, the test is invariant under monotonic transformation of the data which guarantees that no information is lost. Sensoy et al. (2017) apply permutation entropy in testing the efficient market hypothesis on emerging sovereign CDS markets.

Let $\left\{X_{t}\right\}_{t \in I}$ be a real-valued time series. For a positive integer $m \geq 2, S_{m}$ denotes the symmetric group of order $m$ ! (i.e. the group formed by all the permutations of length $m$ ). Let $\pi_{i}=\left(i_{1}, i_{2}, \ldots, i_{m}\right) \in S_{m}$. An element $\pi_{i}$ in the symmetric group $S_{m}$ is called a symbol, and $m$ is usually referred to as the embedding dimension.

Now we define an ordinal pattern for a symbol $\pi_{i}=\left(i_{1}, i_{2}, \ldots, i_{m}\right) \in S_{m}$ at a given time $t \in I$. For this purpose, we consider that the time series is embedded in an $m$-dimensional space as $X_{m}(t)=\left(X_{t+1}, X_{t+2}, \ldots, X_{t+m}\right)$ for $t \in I$. Then, it is said that " $t$ is of $\pi_{i}$ type" if and only if $\pi_{i}=\left(i_{1}, i_{2}, \ldots, i_{m}\right)$ is the unique symbol in the group $S_{m}$ satisfying the two following conditions: (1) $X_{t+i_{1}} \leq X_{t+i_{2}} \leq \cdots \leq X_{t+i_{m}}$ and (2) $i_{s-1} \leq i_{s}$ if $X_{t+i_{s-1}}=X_{t+i_{s}}$. The second condition guarantees uniqueness of the symbol $\pi_{i}$. This is justified if the values of $X_{t}$ have a continuous distribution so that equal values are uncommon, with a theoretical probability of occurrence of 0 .

Notice that for all $t$ such that $t$ is of $\pi_{i}$-type, the $m$-history $X_{m}(t)$ is converted into a unique symbol $\pi_{i}$. This $\pi_{i}$ describes how the ordering of the dates $t+0<t+1<\cdots<t+(m-1)$ is converted into the ordering of the values in the time series under scrutiny.

Also, given a time series $\left\{X_{t}\right\}_{t \in I}$ and an embedding dimension $m$, one could easily compute the relative frequency of a symbol $\pi \in S_{m}$ by

$$
p(\pi):=p_{\pi}=\frac{\#\{t \in I \mid t \text { is of } \pi \text {-type }\}}{|I|-m+1}
$$

where $|I|$ denotes the cardinality of set $I$. Under this setting, the permutation entropy of a time series $\left\{X_{t}\right\}_{t \in I}$ for an embedding dimension $m$ is defined as the Shannon's entropy of the $m$ ! distinct symbols as the following:

$$
h(m)=-\sum_{\pi \in S_{m}} p_{\pi} \ln \left(p_{\pi}\right)
$$

Permutation entropy $h(m)$, is the information contained in comparing $m$ consecutive values of the time series. By definition, $0 \leq h$ $(m) \leq \ln (m !)$ where the lower bound is achieved for monotonic sequence of values, and the upper bound for a completely random system where all $m$ ! possible permutations appear with the same probability. More simply, higher permutation entropy means that the data-generating process is more complex and unpredictable. If a financial time series has a permutation entropy that is significantly low, it implies market inefficiency because the weak-form of market efficiency suggests the unpredictability of future movements for the financial variables (In our analysis, we normalize the permutation entropies (dividing by $\ln (\mathrm{m}$ !)) to achieve a maximum level of 1 ).

To formally test the weak-form efficiency, we use the test of (Matilla-Garcia and Marin, 2008; Lopez et al., 2010). Let $\left\{X_{t}\right\}_{t \in I}$ be a 
real-valued time series with $|I|=T$, and $h(m)$ denotes the permutation entropy of this series for a fixed integer embedding dimension $m>2$ where $m$ is the largest integer that satisfies $5 m ! \leq T$. If $\left\{X_{t}\right\}_{t \in I}$ is independent, then the affine transformation $G(m)=2(T-m+1)(\ln (m !)-h(m))$ is asymptotically $\chi_{m !-1}^{2}$ distributed. Then to test the null hypothesis that $\left\{X_{t}\right\}_{t \in I}$ is independent, the decision rule at $100(1-\alpha) \%$ confidence level is to accept the null hypothesis if $0 \leq G(m) \leq \chi_{m !-1, \alpha}^{2}$, otherwise reject the null hypothesis.

\section{Data and results}

Bitcoin can be traded 24 hours a day and 7 days a week and our data comes from all exchanges where USD and/or EUR can be directly used in Bitcoin trading. ${ }^{2}$ For each trade, the data includes a GMT timestamp, amount of Bitcoin traded and the Bitcoin price in terms of USD or EUR depending on the exchange. We consolidate these trades to get a single trading book. The sample covers all trades from January 1, 2013 to March 5, 2018. Using tick-by-tick trades, we construct the log-return series with 15, 20, 30, 40 and $45 \mathrm{~min}$. frequencies. For each interval, we use the volume-weighted average Bitcoin price (instead of the interval's closing price) to calculate these log-returns.

In our analysis, we choose a weekly time window which is large enough to provide statistical significance. Then we use nonoverlapping rolling windows of weekly intraday data to estimate the permutation entropy and use it to test for independence of returns (we have 270 weeks in total). In this procedure, we call a window "significant" if the null hypothesis of independence is rejected at 0.05 significance level. The rolling-window approach reveals how often the null hypothesis is rejected by the selected test statistic, and hence the percentage of sub-samples with an insignificant test statistic (which we call the efficiency ratio) is used to compare the relative efficiency of the BTCUSD and BTCEUR at different frequencies.

Blue lines in Fig. 1 display the time-varying permutation entropy for each log-returns series. Moreover, to focus on the main trend, we also provide a Hodrick and Prescott (1997) filtered permutation entropy series as shown by the red lines. We see that the Bitcoin market has different degrees of time-varying efficiency depending on the currency and return frequency. For example, efficiency pattern has almost a cyclical behaviour for BTCUSD for any frequency level under consideration. However, as the frequency increases, the up-trend of the cyclical behaviour, starting with the beginning of 2016, reaches up to its maximum levels as of March 2018. This shows that for shorter frequencies, BTCUSD market has become more efficient than ever lately. On the other hand, BTCEUR has a consistent improvement in terms of efficiency throughout the sample period. This improvement, in line with the BTCUSD case, is more emphasized for shorter frequencies with the beginning of 2016. The results on improved efficiency at intraday price levels are in line with the findings of Urquhart (2016) and Bariviera (2017) on the improved efficiency at the daily price levels.

Table 1 presents the efficiency ratios of BTCUSD and BTCEUR for different frequencies. These ratios state that BTCUSD is slightly more efficient than BTCEUR for each frequency in our sample period. This is also validated when we compare the weekly mean permutation entropy levels of BTCUSD and BTCEUR. For each frequency, there is a slight difference in means between these two series.

Table 1 presents another interesting finding regarding informational efficiency and return frequency. Accordingly, regardless of the currency, higher the frequency, lower the informational efficiency is. This might indicate that information is not reflected in prices instantaneously in Bitcoin markets. In our case, the information diffusion process takes some time, i.e., it is spread throughout the consecutive intervals. Since the number of intervals increases when the frequency gets higher, this gradual information diffusion tends to create monotonic return series in a given period.

\subsection{Analysis of standardized returns}

It is widely known that asset returns exhibit volatility clustering most of the time (Rachev et al., 2005). In that case, the existence of the ARCH effect may yield to wrong conclusions when the permutation entropy test is used (Matilla-Garcia and Marin, 2008). In order to overcome this problem, we repeat our main analysis by using GARCH $(1,1)$ filtered returns. In particular, we estimate the following model: $r_{t}=\mu+\varepsilon_{t}$, where $r_{t}=\left[r_{1, t}, \ldots, r_{n, t}\right]^{\prime}$ is the vector of $n$ number of Bitcoin return series in our sample, $\mu$ is a vector of constants with length $n$, and $\varepsilon_{t}=\left[\varepsilon_{1, t}, \ldots, \varepsilon_{n, t}\right]^{\prime}$ is the vector of residuals. Following that, conditional volatilities $h_{i, t}$ are obtained from univariate $\operatorname{GARCH}(1,1)$ process $h_{i, t}^{2}=\omega+\alpha \varepsilon_{i, t-1}^{2}+\beta h_{i, t-1}^{2}$. In this part, we implement the permutation entropy methodology on the standardized residuals $u_{i, t}=\varepsilon_{i, t} / h_{i, t}$. The last columns in Table 1 show the efficiency ratios and mean permutation entropy levels based on the standardized return series. Although there are slight changes in the efficiency ratios and mean permutation entropy levels, all of our previous findings still hold.

\subsection{Determinants of efficiency}

In this part, we seek potential determinants of pricing efficiency in Bitcoin markets. Two important market variables, namely liquidity and volatility, are considered. For each return series, permutation entropy levels in a week are taken as the weekly efficiency measure. As a proxy for liquidity (LIQ), we take USD (EUR) trading volume for BTCUSD (BTCEUR) in the corresponding week.

\footnotetext{
${ }^{2}$ These exchanges are 1coin, abucoins, allcoin, aqoin, anxhk, bitbay, bitkonan, bitstamp, btcalpha, btcc, b2c, b7, bcmBM, bcmLR, bcmMB, bcmPP, bitalo, bitbox, bitcurex, bitfinex, bitfloor, bitmarket, bitme, btc24, btce, btcex, btcexWMZ, btctree, bc, btcde, btceur, coinfalcon, cex, coinbase, coinsbank, cbx, cotr, cryptox, crytr, exchb, exmo, fbtc, global, hitbtc, itbit, ibwt, imcex, indacoin, intrsng, just, kraken, lake, localbtc, lybit, mtgox, okcoin, ripple, rock, ruxum, thLR, th, vcx, weex, and zyado.
} 

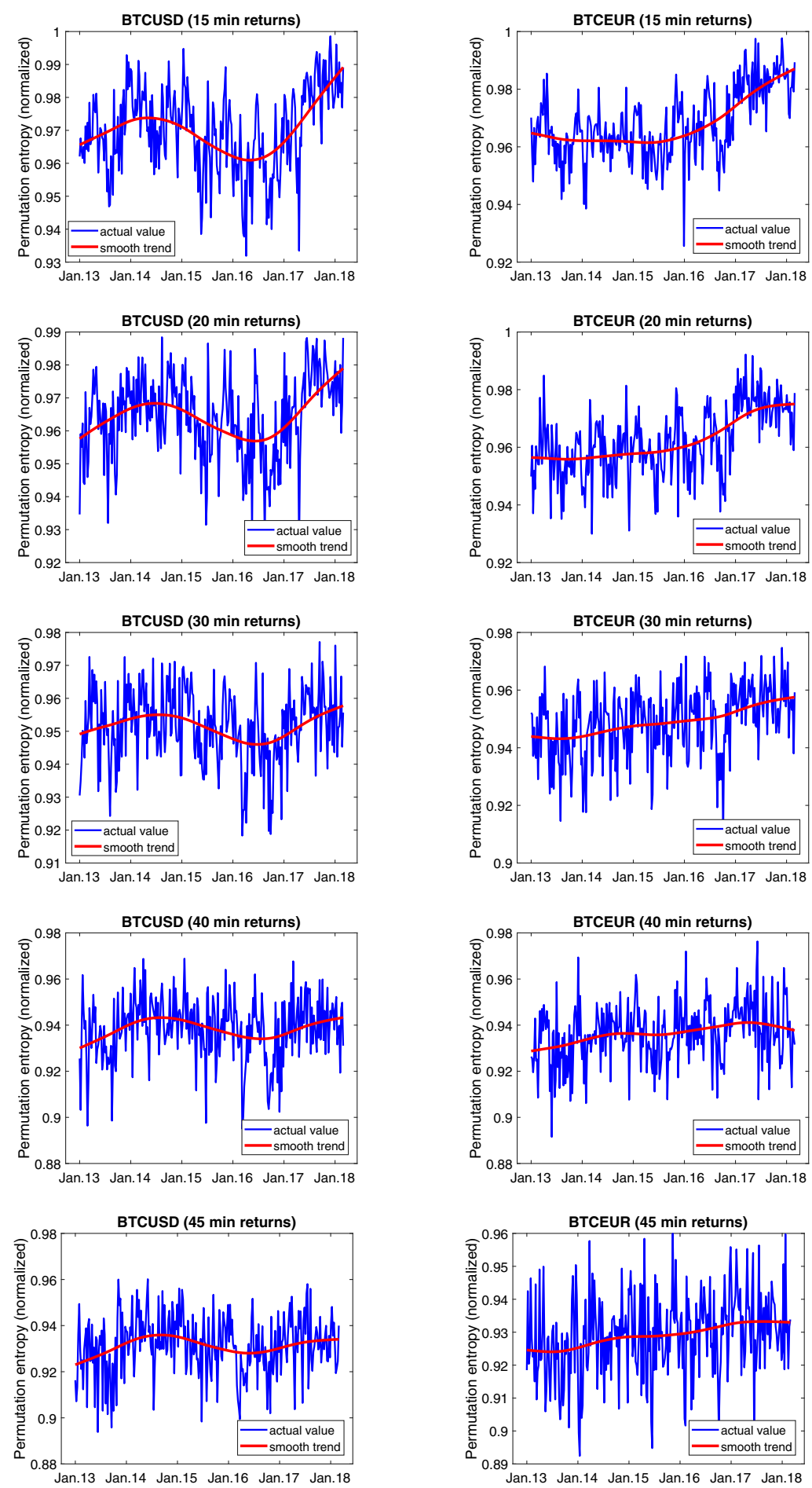

Fig. 1. Time varying normalized permutation entropies for BTCUSD and BTCEUR log-return series with different frequencies. The red lines in the figures are smoothed trends estimated by applying Hodrick-Prescott filter on weekly permutation entropy series. (For interpretation of the references to colour in this figure legend, the reader is referred to the web version of this article.)

Finally, standard deviation of the returns in that week is taken as the volatility proxy $(V O L)$. We estimate the following regression in Eq. (1) to carry out our analysis:

$$
\Delta P E_{t}=\alpha+\beta_{1} \Delta L I Q_{t}+\beta_{2} \Delta V O L_{t}+\epsilon_{t}
$$


Table 1

The efficiency ratios of Bitcoin markets based on log-returns and $\operatorname{GARCH}(1,1)$ filtered returns, and their corresponding mean permutation entropy levels.

\begin{tabular}{|c|c|c|c|c|c|c|c|c|}
\hline \multirow[b]{2}{*}{ Return frequency } & \multicolumn{2}{|c|}{ Efficiency ratio } & \multicolumn{2}{|l|}{ Mean PE } & \multicolumn{2}{|c|}{ Efficiency ratio (GARCH) } & \multicolumn{2}{|c|}{ Mean PE (GARCH) } \\
\hline & BTCUSD & BTCEUR & BTCUSD & BTCEUR & BTCUSD & BTCEUR & BTCUSD & BTCEUR \\
\hline $15 \mathrm{~min}$. & 0.330 & 0.244 & 0.970 & 0.968 & 0.196 & 0.167 & 0.968 & 0.966 \\
\hline $20 \mathrm{~min}$. & 0.367 & 0.278 & 0.964 & 0.962 & 0.267 & 0.211 & 0.962 & 0.960 \\
\hline $30 \mathrm{~min}$. & 0.426 & 0.374 & 0.952 & 0.949 & 0.322 & 0.300 & 0.950 & 0.947 \\
\hline $40 \mathrm{~min}$. & 0.530 & 0.437 & 0.938 & 0.936 & 0.437 & 0.378 & 0.936 & 0.935 \\
\hline $45 \mathrm{~min}$. & 0.537 & 0.444 & 0.931 & 0.929 & 0.489 & 0.426 & 0.930 & 0.929 \\
\hline
\end{tabular}

Table 2

Parameter estimates for the determinants of efficiency.

\begin{tabular}{|c|c|c|c|}
\hline$\triangle P E_{t}$ & $\alpha$ & $\triangle L I Q_{t}$ & $\triangle V O L_{t}$ \\
\hline BTCUSD (15 min) & $\begin{array}{l}-0.0004 \\
(-0.57)\end{array}$ & $\begin{array}{l}0.0121 * * * \\
(8.40)\end{array}$ & $\begin{array}{l}-0.0067^{* * *} \\
(-2.67)\end{array}$ \\
\hline BTCEUR (15 min) & $\begin{array}{l}-0.0000 \\
(-0.01)\end{array}$ & $\begin{array}{l}0.008^{* * *} \\
(4.74)\end{array}$ & $\begin{array}{l}-0.0018 \\
(-0.82)\end{array}$ \\
\hline BTCUSD (20 min) & $\begin{array}{l}-0.0003 \\
(-0.41)\end{array}$ & $\begin{array}{l}0.0119 * * * \\
(6.54)\end{array}$ & $\begin{array}{l}-0.0051 * \\
(-1.67)\end{array}$ \\
\hline BTCEUR (20 min) & $\begin{array}{l}-0.0003 \\
(-0.47)\end{array}$ & $\begin{array}{l}0.0096 * * * \\
(5.10)\end{array}$ & $\begin{array}{l}-0.0074 * * * \\
(-3.22)\end{array}$ \\
\hline BTCUSD (30 min) & $\begin{array}{l}-0.0001 \\
(-0.13)\end{array}$ & $\begin{array}{l}0.0133^{* * *} \\
(6.97)\end{array}$ & $\begin{array}{l}-0.0078 * * * \\
(-2.68)\end{array}$ \\
\hline BTCEUR (30 min) & $\begin{array}{l}-0.0006 \\
(-0.64)\end{array}$ & $\begin{array}{l}0.0143^{* * *} \\
(5.40)\end{array}$ & $\begin{array}{l}-0.009 * * * \\
(-3.02)\end{array}$ \\
\hline BTCUSD (40 min) & $\begin{array}{l}-0.0004 \\
(-0.37)\end{array}$ & $\begin{array}{l}0.0087 * * * \\
(3.08)\end{array}$ & $\begin{array}{l}-0.0028 \\
(-0.68)\end{array}$ \\
\hline BTCEUR (40 min) & $\begin{array}{l}0.0001 \\
(0.06)\end{array}$ & $\begin{array}{l}0.0154 * * * \\
(5.00)\end{array}$ & $\begin{array}{l}-0.0136 * * * \\
(-4.19)\end{array}$ \\
\hline BTCUSD (45 min) & $\begin{array}{l}-0.0001 \\
(-0.09)\end{array}$ & $\begin{array}{l}0.0092^{* * *} \\
(3.26)\end{array}$ & $\begin{array}{l}-0.0073^{*} \\
(-1.90)\end{array}$ \\
\hline BTCEUR (45 min) & $\begin{array}{l}-0.0004 \\
(-0.39)\end{array}$ & $\begin{array}{l}0.0108^{* * *} \\
(3.31)\end{array}$ & $\begin{array}{l}0.0002 \\
(0.06)\end{array}$ \\
\hline
\end{tabular}

1. The values in the parentheses are t-stats obtained from robust standard errors.

$2 . *, * *$ and $* * *$ denote significance at $10 \%, 5 \%$ and $1 \%$ levels respectively.

In Eq. (1), $\triangle P E_{t}$ refers to the log-return of permutation entropy from week $t-1$ to $t$, i.e., $\ln \left(P E_{t} / P E_{t-1}\right)$. Similarly, $\triangle L I Q_{t}$ and $\triangle V O L_{t}$ denote the weekly log-returns of the relevant variables. Estimation is performed via robust ordinary least squares with bisquare algorithm. Table 2 presents the estimated parameters.

According to Table 2, liquidity has a strong influence on the weak-form efficiency. For any return series, an increase in liquidity comes with an improved pricing efficiency, which supports the findings by Brauneis and Mestel (2018). On the contrary, volatility has a significant negative impact on the efficiency. Although the significance is not as strong as in the case of liquidity, a decrease in volatility tend to create a higher informationally efficient market for Bitcoin prices.

\section{Conclusion}

The analysis above is the first to study the weak-form efficiency of the Bitcoin prices at high-frequency and in terms of euro in addition to the US dollar. We employ permutation entropy with a rolling window approach to test for the weak-form of market efficiency of intraday Bitcoin prices. There are four principal findings.

First, BTCUSD and BTCEUR markets have become more informationally efficient at the intraday level since the beginning of 2016, supporting the findings of Urquhart (2016) and Bariviera (2017) on the lately improved informational efficiency at the daily frequency. However, according to our findings, this improvement has a cyclical pattern for BTCUSD, whereas it is a gradual increase for BTCEUR. Second, BTCUSD market is slightly more efficient than BTCEUR market in the sample period, which might indicate that BTCEUR markets provide a more profitable trading environment at the intraday level. Third, regardless of the currency choice, higher the frequency, lower the pricing efficiency is. Fourth, liquidity (volatility) is found to have a significant positive (negative) effect on the informational efficiency of Bitcoin prices.

\section{References}

Al-Yahyaee, K.H., Mensi, W., Yoon, S.M., 2018. Efficiency, multifractality, and the long-memory property of the bitcoin market: a comparative analysis with stock, 
currency, and gold markets. Finance Res. Lett. http://dx.doi.org/10.1016/j.frl.2018.03.017.

Bandt, C., Pompe, B., 2002. Permutation entropy - a natural complexity measure for time series. Phys. Rev. Lett. 88, 174102.

Bariviera, A., 2017. The inefficiency of bitcoin revisited: a dynamic approach. Econ. Lett. 161, 1-4.

Brauneis, A., Mestel, R., 2018. Price discovery of cryptocurrencies: bitcoin and beyond. Econ. Lett. 165, 58-61.

Corbet, S., Meegan, A., Larkin, C., Lucey, B., Yarovaya, L., 2018. Exploring the dynamic relationships between cryptocurrencies and other financial assets. Econ. Lett. $165,28-34$.

Demir, E., Gozgor, G., Lau, C.K.M., Vigne, S.A., 2018. Does economic policy uncertainty predict the bitcoin returns? an empirical investigation. Finance Res. Lett. http://dx.doi.org/10.1016/j.frl.2018.01.005.

Fama, E.F., 1970. Efficient capital markets: a review of theory and empirical work. J. Finance 25, 383-417.

Fry, J., Cheah, E.T., 2015. Speculative bubbles in bitcoin markets? an empirical investigation into the fundamental value of bitcoin. Econ. Lett. 130, 32-36.

Glantz, M., Kissell, R., 2013. Multi-Asset Risk Modeling: Techniques for a Global Economy in an Electronic and Algorithmic Trading Era. Academic Press.

Hodrick, R., Prescott, E.C., 1997. Postwar U.S. business cycles: an empirical investigation. J. Money Credit Bank. 29, 1-16.

Lopez, F., Matilla-Garcia, M., Mur, J., Marin, M.R., 2010. A non-parametric spatial independence test using symbolic entropy. Reg. Sci. Urban Econ. 40, 106-115. Matilla-Garcia, M., Marin, M.R., 2008. A non-parametric independence test using permutation entropy. J. Econom. 144, 139-155.

Nadaraj, S., Chu, J., 2017. On the inefficiency of bitcoin. Econ. Lett. 150, 6-9.

Nakamoto, S., 2008. Bitcoin: a peer-to-peer electronic cash system.

Panagiotidis, T., Stengos, T., Vravosinos, O., 2018. On the determinants of bitcoin returns: a LASSO approach. Finance Res. Lett. http://dx.doi.org/10.1016/j.frl.2018. 03.016.

Rachev, S.T., Menn, C., Fabozzi, F.J., 2005. Fat-tailed and Skewed Asset Return Distributions: Implications for Risk Management, Portfolio Selection, and Option Pricing. Wiley, Hoboken, NJ.

Sensoy, A., Aras, G., Hacihasanoglu, E., 2015. Predictability dynamics of islamic and conventional equity markets. N. Am. J. Econ. Finance 31, 222-248.

Sensoy, A., Fabozzi, F.J., Eraslan, V., 2017. Predictability dynamics of emerging sovereign CDS markets. Econ. Lett. 161, 5-9.

Tiwari, A.K., Jana, R.K., Das, D., Roubaud, D., 2018. Informational efficiency of bitcoin: an extension. Econ. Lett. 163, 106-109.

Urquhart, A., 2016. The inefficiency of bitcoin. Econ. Lett. 148, 80-82.

Vidal-Tomas, D., Ibanez, A., 2018. Semi-strong efficiency of bitcoin. Finance Res. Lett. http://dx.doi.org/10.1016/j.frl.2018.03.013.

Yonghong, J., He, N., Weiuha, R., 2018. Time-varying long-term memory in bitcoin market. Finance Res. Lett. http://dx.doi.org/10.1016/j.frl.2017.12.009. 